\title{
Title: efficacy of intravitreal dexamethasone implant on hard exudate in diabetic macular edema
}

Chang Ki Yoon', Min Sagong ${ }^{2}$, Jae Pil Shin ${ }^{3}$, Sang Joon Lee ${ }^{4}$, Joo Eun Lee ${ }^{5}$, Ji Eun Lee ${ }^{6}$, Inyoung Chung ${ }^{7}$, Woo Jin Jeong ${ }^{8}$, Kang Yeun Pak ${ }^{9}$ and Hyun Woong Kim ${ }^{9,10^{*}}$ (1)

\begin{abstract}
Background: To investigate the effect of intravitreal dexamethasone implant (DEX implant) on hard exudate (HE) accompanying diabetic macular edema (DME).

Methods: This study was a non-comparative non-randomized 1-year prospective interventional study. Patients with DME and HE were treated using DEX implant two or three times. Color fundus photography and optical coherence tomography $(\mathrm{OCT})$ were performed at every visit. HE area was measured semi-automatically from the fundus photographs.

Results: Thirty-five patients completed the study. Eleven patients (31.4\%) received two injections, while the remaining received three times. HE area (primary outcome) significantly decreased from $1.404 \pm 2.094 \mathrm{~mm} 2$ (baseline) to $0.212 \pm 0.592 \mathrm{~mm} 2$ (last visit), which was $24 \%$ of the baseline HE area $(P<0.001)$. HE1500 (HE within 1500 $\mu \mathrm{m}$ from the fovea) area also decreased significantly from $0.382 \pm 0.467 \mathrm{~mm} 2$ to $0.066 \pm 0.126 \mathrm{~mm} 2(P<0.001)$. Furthermore, anaverage best corrected visual acuity (BCVA) improvement of 4.4 Early Treatment Diabetic Retinopathy Study (ETDRS) letters was observed (from $49.9 \pm 18.3$ to $54.3 \pm 20.4$ letters) ( $P=0.008$ ). Central macular thickness (CMT) decreased from $455.8 \pm 23.6 \mu \mathrm{m}$ to $366.8 \pm 31.1 \mu \mathrm{m}(P=0.009)$. Repetitive measurements for entire study duration was analyzed using generalized estimating equations (GEE), where BCVA was related to age, CMT, and HE1500 area in multivariate analyses.
\end{abstract}

Conclusion: DEX implant could reduce and suppress HE in DME for one year with two or three injections. And centrally located HE area (HE1500 area) is related to vision.

Trial registration: ClinicalTrials.gov, NCT02399657, Registered 26 March 2015.

Keywords: Dexamethasone, Diabetic retinopathy, Exudate, Intravitreal injection, Macular edema

\footnotetext{
* Correspondence: maekbak@gmail.com

${ }^{9}$ Inje Univertisy, Haeundae Paik hospital, 875, Haeun-daero, Haeundae-gu, 48108 Busan, Korea

${ }^{10}$ Inje University Pusan Paik hospital, 875, Haeun-daero, Haeundae-gu, 48108 Busan, Korea

Full list of author information is available at the end of the article
}

(C) The Author(s). 2021 Open Access This article is licensed under a Creative Commons Attribution 4.0 International License, which permits use, sharing, adaptation, distribution and reproduction in any medium or format, as long as you give appropriate credit to the original author(s) and the source, provide a link to the Creative Commons licence, and indicate if changes were made. The images or other third party material in this article are included in the article's Creative Commons licence, unless indicated otherwise in a credit line to the material. If material is not included in the article's Creative Commons licence and your intended use is not permitted by statutory regulation or exceeds the permitted use, you will need to obtain permission directly from the copyright holder. To view a copy of this licence, visit http://creativecommons.org/licenses/by/4.0/ The Creative Commons Public Domain Dedication waiver (http://creativecommons.org/publicdomain/zero/1.0/) applies to the data made available in this article, unless otherwise stated in a credit line to the data. 


\section{Background}

Diabetic macular edema (DME) is a major visionthreatening complication of diabetes mellitus. The overall prevalence of DME is estimated to be $6.96 \%$ in diabetic patients, with a cumulative incidence of approximately $25 \%$ in type 2 diabetes patients treated with insulin $[1,2]$. DME has a poor prognosis if untreated, and eyes with DME involving the fovea showed moderate vision loss in $29 \%$ and visual recovery of 3 Early Treatment Diabetic Retinopathy Study (ETDRS) lines in only $5 \%$ of individuals [3]. Several clinical trials have demonstrated that intravitreal anti-vascular endothelial growth factor (anti-VEGF) injection reduces vision loss and even improves vision [4-6]. Intravitreal steroid injection has also been proved to be effective in DME treatment [7].

Hard exudate (HE) often accompanies DME. HE, which presents yellow-white deposits in retina, is thought to consist of lipid exudation. While HE can occasionally resolve spontaneously, it can form fibrotic lesions that lead to severe vision loss. $\mathrm{HE}$ is related to poor visual outcomes in patients with DME [8, 9]. Especially, subfoveal HE was demonstrated to be an independent risk factor for visual decline [10]. Several studies have shown HE resolution after using anti-VEGF or intravitreal dexamethasone (DEX) implant $[9,11,12]$. Some researchers showed that the steroid is superior to anti-VEGF agents [11]. In addition, DEX implant can be advantageous in that it stays for a longer duration in the eye. However, aforementioned studies had limitations regarding their short study durations and post hoc analyses in which $\mathrm{HE}$ was not the primary outcome of interest. Moreover, they showed conflicting outcomes about $\mathrm{HE}$ reduction. Therefore, we investigated the effect of DEX implant on HE in DME in a prospective cohort.

\section{Methods}

Patients with DME and HE were recruited from 8 tertiary medical centers. DME was defined as macular thickening resulting from diabetic retinopathy and not by another cause. Cases were included if baseline central macular thickness (CMT) exceeded $300 \mu$ mand had visible $\mathrm{HE}$ within $1500 \mu \mathrm{m}$ to the fovea. Additionally, patients were included if their vision ranged from $20 / 320$ to $20 / 40$. If both eyes were eligible, the eye with greater $\mathrm{HE}$ was selected. The main exclusion criteria were as follows: concurrent retinal disease that may provoke macular edema, intraocular surgery or intravitreal anti-VEGF injection within 3 months of study entry, intravitreal or subconjunctival steroid injection within 6 months of study entry, eyes with media opacity hindering imaging study required for study protocol, uncontrolled systemic disease, patients requiring systemic glucocorticoid or immunosuppressant treatment and patients not suitable for DEX implant. All study conduct adhered to the tenets of the Declaration of Helsinki, and the study protocol was approved by the Institutional Review Board.

All study eyes received DEX implant (Ozurdex; Allergan, Irvine, California, USA) right after screening evaluation (first injection) and at 4 or 5 month-visit (second injection). Additionally, from 8 to 11 months, the study eye could receive a third injection as to investigator's determination based on the CMT exceeding $350 \mu \mathrm{m}$ or exacerbation of vision more than 5 letters compared to best vision. Study visits were scheduled every month until 12 months following study initiation. Best corrected visual acuity (BCVA) measured using ETDRS chart, intraocular pressure (IOP), slit lamp examination, and fundus examination were performed every visit. Standard fundus photography and optical coherence tomography (OCT) (Cirrus OCT: Carl Zeiss Meditec, Dublin, CA or Spectralis OCT: Heidelberg Engineering, Heidelberg, Germany) were performed at every visit. Complete blood cell count, basic chemistry analysis including HbA1c and lipid profiles were measured at screening and final visit. Fluorescein angiography and indocyanine-green angiography were performed at baseline and final visit. Cataract was graded according to lens opacification classification system (LOCS III grading). Each investigator separately graded the cataract status.

Macular HE area within $1500 \mu \mathrm{m}$ from the fovea center (HE1500 area) and within arcade (4000 $\mu \mathrm{m}$ from the fovea center, HE area) were measured from fundus photograph semi-automatically using ImageJ software (Rasband, W.S., Image), U. S. National Institutes of Health, Bethesda, Maryland, USA). Two masked graders (GCJ and JHL) performed the analysis individually. Reference scale and fovea location were obtained from overlay of OCT image on fundus photo. OCT report image contains retinal image displaying scanned area and foveola. Thus, we are able to get actual scale of the fundus photo and foveola location by superimposing the OCT retina image on color fundus photo. After uploading fundus photography into ImageJ, green channel image was used for further processing. Basically, binary images separating HE were generated by the automatic threshold function of ImageJ and manual adjusting. In the binary image, pixels other than $\mathrm{HE}$, including vessel or optic disc, was erased manually. Next, the total area of HE within $4000 \mu \mathrm{m}$ and $1500 \mu \mathrm{m}$ circle were measured (Fig. 1). Interclass correlation between the two graders was $0.95(\mathrm{P}<0.001)$. The average value of $\mathrm{HE}$ area identified by the two graders was used for statistical analysis. 


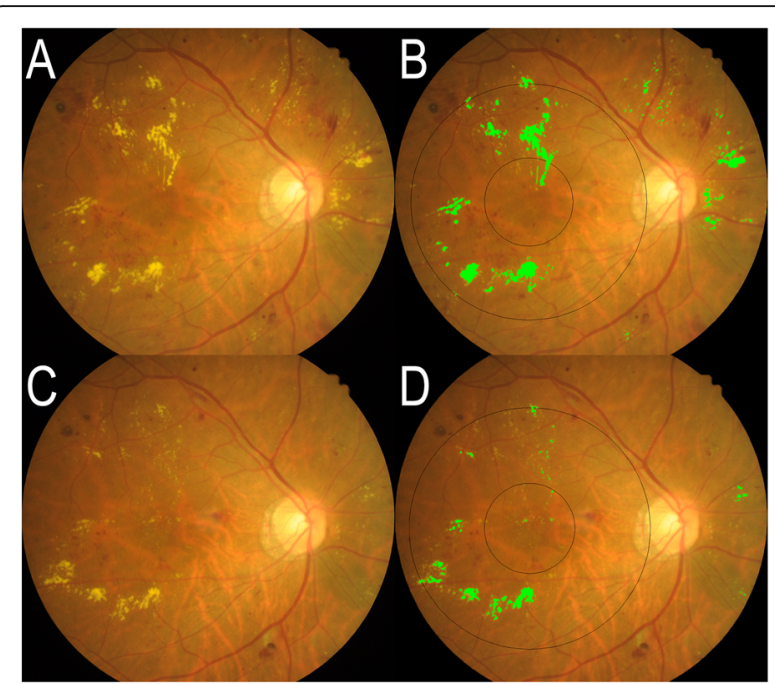

Fig. 1 Fundus photograph and measurement of hard exudate in a representative case. $\mathbf{a}$ and $\mathbf{c}$ Fundus photograph of the same patient at baseline and last visit, respectively. $\mathbf{b}$ and $\mathbf{d}$ Hard exudate (HE) area analysis in $\mathbf{a}$ and $\mathbf{c}$. Light green colored pixels indicate the identified HE. Vague tiny yellow particles are not marked as HE. Black circles are $3 \mathrm{~mm}$ and $8 \mathrm{~mm}$ diameter circles centered at the fovea. These circles are generated using superimposed optical coherence tomography fundus image as reference. HE area and HE1500 area are defined as the area of HE within $8 \mathrm{~mm}$ and $3 \mathrm{~mm}$ circle, respectively
The presence of subfoveal fluid at initial examination was determined using OCT. Morphologic typing of macular edema as cystoid macular edema or diffuse retinal thickening followed the classification of Kim et al. [13]. Microaneurysm number was counted from fluorescein angiogram [14].

Paired t-tests were used to compare the change of variables from the initial value at each time point. (Table 1; Figs. 2 and 3) Linear regression analysis was used to determine the factors that influenced initial vision and $\mathrm{HE}$ area. HE area was log-transformed to be normalized in linear regression analysis. For analyzing the repetitive measurements, generalized estimating equation (GEE) models were generated for univariable and multivariable analyses to estimate the relationships of outcome variables with age, sex, routine laboratory data, cataract progression, event of increased IOP and the number of Dex injections. Potential relating variables with a $\mathrm{P}$ value of $\leq 0.10$ on univariate analysis were tested on multivariable analysis. An autoregressive correlation structure was assumed in these models to account for the within subject correlation. Statistical analyses were performed using the software package SPSS 23.0 (IBM Corp. Released 2015. IBM SPSS Statistics for Windows, Version 23.0. Armonk, NY: IBM Corp.). A P value of $<0.05$ was considered statistically significant. Sample size was determined as 48 based on following parameters. Proportion of patients who showed reduction of HE was estimated to be 0.9 , significance level was 0.05 , margin of error was 0.1 and drop-out rate was 0.25 .

The study protocol followed the guidelines of the Declaration of Helsinki. Written informed consent was

Table 1 Characteristics of study populations

\begin{tabular}{|c|c|c|c|}
\hline & Baseline values & After 1 year values & $\mathrm{P}^{*}$ \\
\hline$\overline{\text { Age }(\text { Avg }[S D])^{\dagger}, \text { years }}$ & $59.3(9.9)$ & & \\
\hline Male: Female & $12: 23$ & & \\
\hline Hypertension & $12(34.3 \%)$ & & \\
\hline CKD & $2(5.6 \%)$ & & \\
\hline Dyslipidemia & $7(19.4 \%)$ & & \\
\hline HbA1c (Avg [SD]) & $7.96(1.61) \%$ & $7.56(1.22) \%$ & 0.105 \\
\hline BUN (Avg [SD]) & $18.1(5.2) \mathrm{mg} / \mathrm{dl}$ & $20.4(7.6) \mathrm{mg} / \mathrm{dl}$ & 0.052 \\
\hline $\mathrm{Cr}(\mathrm{Avg}[\mathrm{SD}])$ & $1.02(1.19) \mathrm{mg} / \mathrm{dl}$ & $1.19(0.99) \mathrm{mg} / \mathrm{dl}$ & 0.073 \\
\hline TG (Avg [SD]) & $171.0(77.2) \mathrm{mg} / \mathrm{dl}$ & $148.9(82.6) \mathrm{mg} / \mathrm{dl}$ & 0.122 \\
\hline Total cholesterol (Avg [SD]) & $183.8(53.5) \mathrm{mg} / \mathrm{dl}$ & $170.4(39.0) \mathrm{mg} / \mathrm{dl}$ & 0.222 \\
\hline $\mathrm{HDL}$ (Avg [SD]) & $50.0(15.0) \mathrm{mg} / \mathrm{dl}$ & $50.7(12.0) \mathrm{mg} / \mathrm{dl}$ & 0.859 \\
\hline $\operatorname{LDL}(\operatorname{Avg}[S D])$ & $120.4(52.0) \mathrm{mg} / \mathrm{dl}$ & $100.8(37.1) \mathrm{mg} / \mathrm{dl}$ & $0.048^{\ddagger}$ \\
\hline diffuse: focal edema & $16(45 \%): 19(55 \%)$ & & \\
\hline Presence of SRF & $11(31 \%)$ & & \\
\hline Microaneurysm count (Avg [SD]) & $15.3(13.3)$ & $13.1(10.5)$ & $0.032^{\ddagger}$ \\
\hline
\end{tabular}

CKD chronic kidney disease, Presence of SRF presence of macular subretinal fluid, BUN blood urea nitrogen, $C r$ creatinine, $T G$ triglyceride, $H D L$ high density lipoprotein, $L D L$ low density lipoprotein

*paired t-test; $\uparrow$ Avg [SD]: average [standard deviation]; $¥ P<0.05$ 


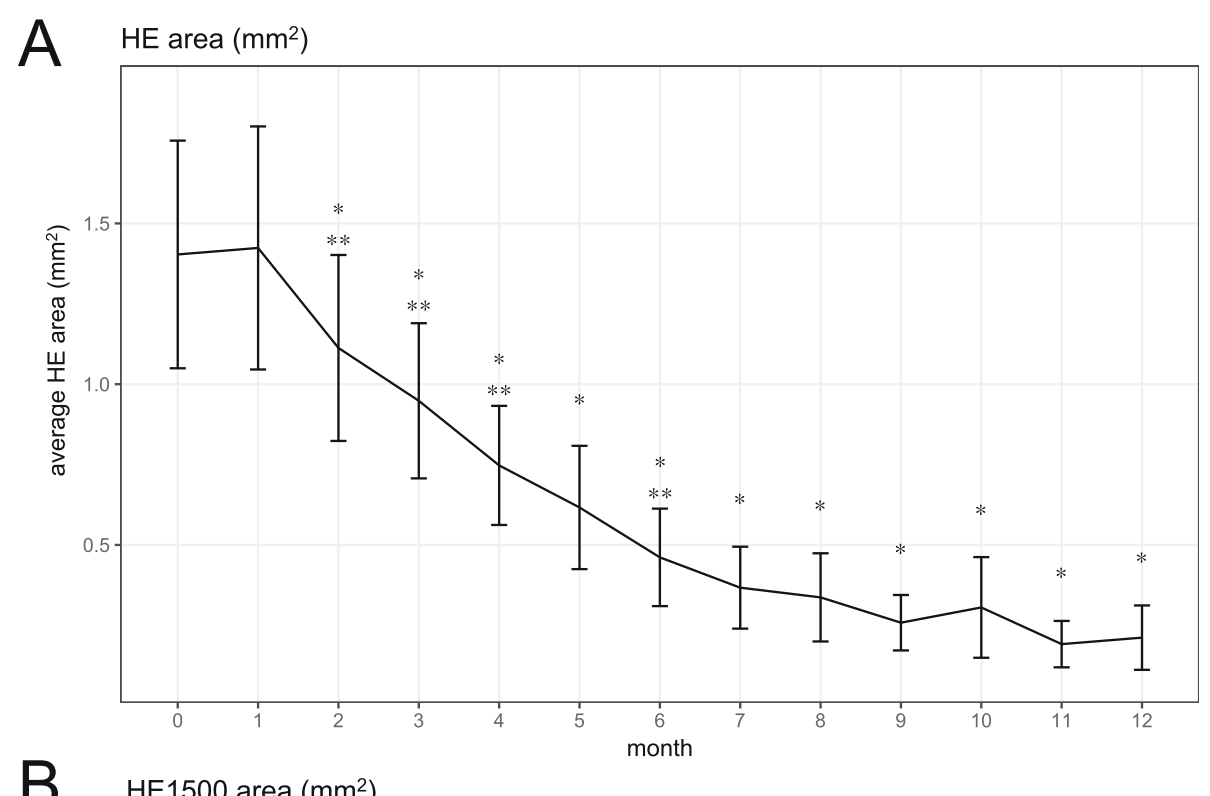

B HE1500 area $\left(\mathrm{mm}^{2}\right)$

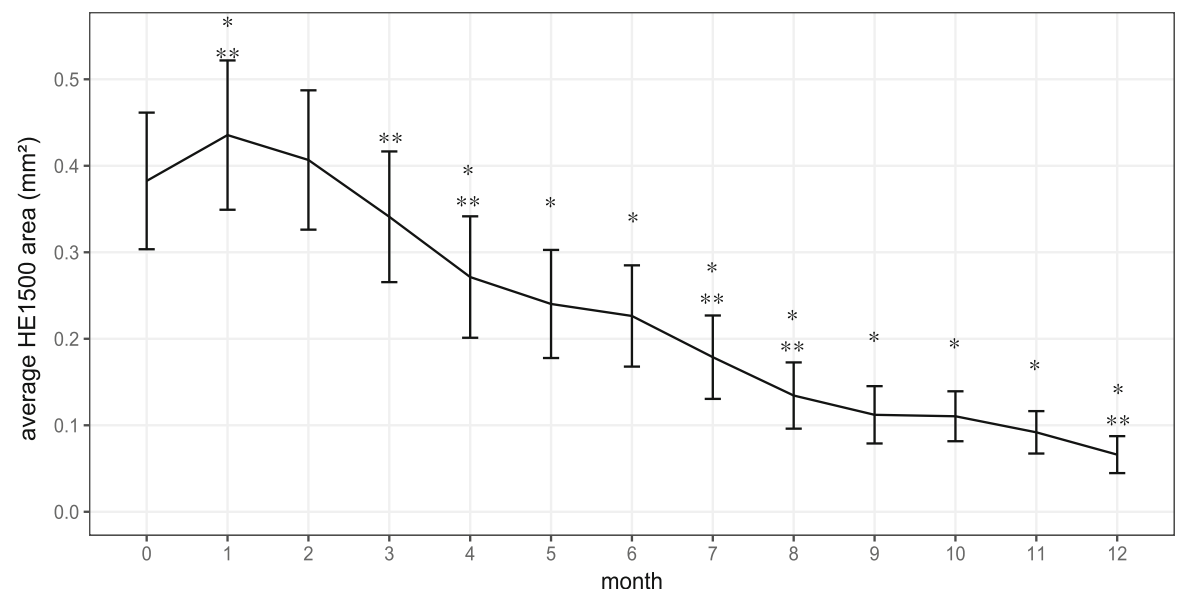

Fig. 2 Line plots of hard exudate (HE) area. a Line plot of the average HE area of each visit. b Line plot of the average HE1500 area. HE1500: HE within $1500 \mu \mathrm{m}$ from the fovea. ${ }^{*} p<0.05$, paired t-test compared to baseline value, ${ }^{* *} p<0.05$, paired t-test compared to value of 1 month ago

obtained from all of the participants before the study began.

\section{Results}

Forty-eight patients were initially enrolled. Four patients withdrew consent, one patient failed to pass screening, one patient underwent vitrectomy due to vitreous hemorrhage, one patient was hospitalized for chronic kidney disease aggravation and six patients were lost during follow up. Finally, 35 patients completed the study protocol. (Fig. 4) The two required injections were done in all the participants and additional third injection was done in 24 patients (68.6\%) depending on investigator's decision. The mean age was 59.3 years (range 29 to 73, standard deviation (SD) 9.8) and 12 (34.3\%) were male. Although study protocol allowed for laser treatment as a rescue therapy after 4 months from first injection, no patients underwent laser treatment. Among blood chemistry analysis, low density lipoprotein (LDL) cholesterol decreased significantly after one year, and microaneurysm count located within $3000 \mu \mathrm{m}$ of the fovea decreased. Detailed baseline characteristics and blood chemistry are presented in Table 1 .

Average HE area (SD) decreased from $1.403(2.094) \mathrm{mm}^{2}$ at baseline to $0.211(0.592) \mathrm{mm}^{2}$ at final visit $(P<0.001$, paired t-test), which was $26 \%$ of the total baseline area. Mean HE1500 area (SD) also decreased significantly from $0.382(0.467) \mathrm{mm}^{2}$ at baseline to $0.066(0.126) \mathrm{mm}^{2}$ at final visit $(P<0.001$, paired $\mathrm{t}$-test), a decrease to $17 \%$ of the initial total HE1500 area. Among the 33 patients who had an 

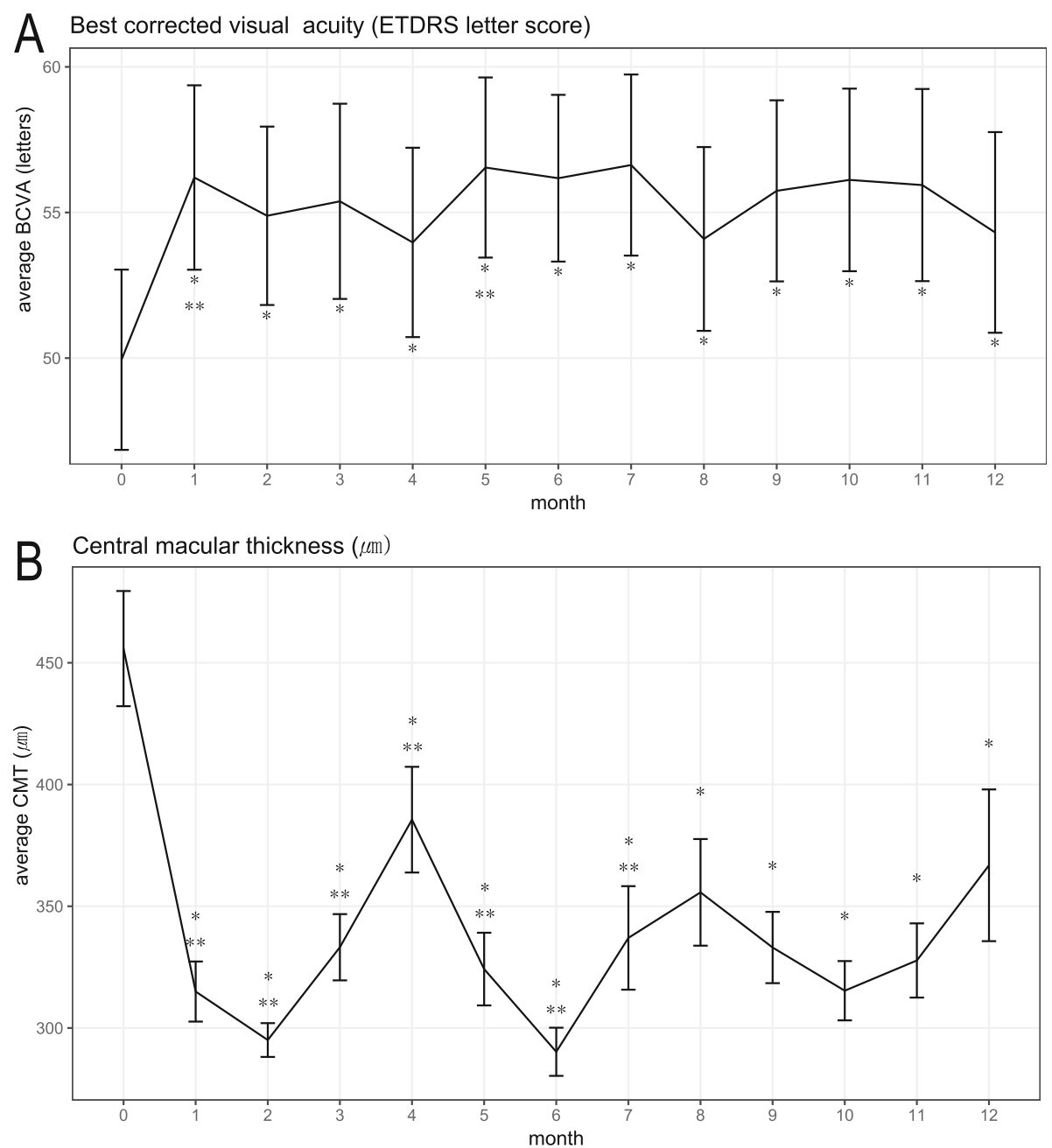

Fig. 3 Line plots of average BCVA and CMT (a) Line plot of the average best corrected visual acuity (BCVA) measured by Early Treatment Diabetic Retinopathy Study (ETDRS) chart. b Line plot of the average central macular thickness (CMT). ${ }^{*} p<0.05$, paired t-test compared to baseline value, ** $p<0.05$, paired t-test compared to value of 1 month ago

HE1500 larger than $0.001 \mathrm{~mm}^{2}$ measured by photo analysis at baseline, HE1500 area decreased in $26(79 \%)$ patients at 10 months and in 28 (85\%) by final visit (Fig. 2). HE area significantly decreased from month 2 and HE1500 area decreased from month 3 . HE1500 area increased significantly at month 1. $\left(0.382 \mathrm{~mm}^{2}\right.$ to $\left.0.435 \mathrm{~mm}^{2}, P=0.009\right)$

The improvement of average BCVA (SD) was 4.4 (9.3) letters (average BCVA [SD] were 49.9 [18.3] letters at baseline and $54.3[20.3]$ letters at 1 year $[P=0.008$, paired t-test]). Average CMT (SD) decreased significantly from $455.8(23.6) \mu \mathrm{m}$ at baseline to 366.8 (31.1) $\mu \mathrm{m}$ at 1 year $(P=0.009$, paired t-test) (Fig. 3$)$. The reduction of average CMT (SD) was $89(191.5) \mu \mathrm{m}$. (Fig. 3) The proportion of patients whose CMT was under $290 \mu \mathrm{m}$ was $34.2 \%$ (12 out of 35) at last visit. HE area reduction (Initial HE area - final HE area) showed positive correlation with BCVA improvement (Final BCVA - Initial BCVA) (Pearson correlation coefficient $=0.161, P<$
0.001). However, there was no correlation between HE and CMT reduction.

Baseline BCVA was related to initial HbA1c. Baseline HE area was related to total cholesterol, LDL level, and microaneurysm count in the univariable analysis. Microaneurysm count was only significantly correlated with $\mathrm{HE}$ area in the multivariable analysis. (Table 2)

The generalized estimating equation (GEE) was generated where repetitive measurements of $\mathrm{HE}$ area or BCVA in one person over 1 year were designated as outcome variables and visit month was a within variable. When BCVA was outcome variable, initial HbA1c, initial microaneurysm count, age and repetitive measurements of CMT, HE area, HE1500 area were significantly correlated to BCVA. In multivariable model, age, HE1500 area and CMT were statistically meaningful parameters (Table 3). However, when HE area was assigned as outcome variable, neither parameters in Table 3 showed 


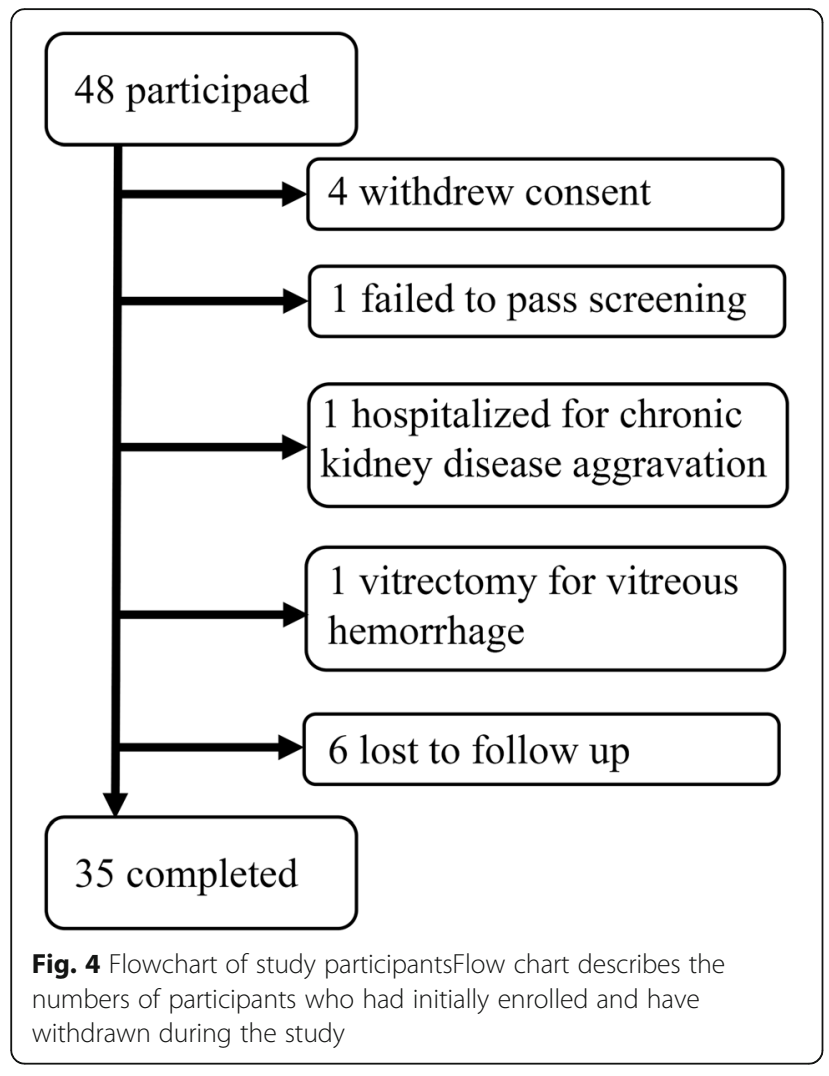

any statistical relationships with $\mathrm{HE}$ area. (HE area and HE 1500 area were omitted in this analysis).

Cataract progression was observed in 16 out of 35 (45.7\%) patients. LOCS grading revealed aggravated nuclear opacity (37.1\%) and post-subcapsular opacity (20.0\%). Two patients underwent cataract surgery during the study duration. IOP exceeding $25 \mathrm{mmHg}$ at any time was observed in five patients (11\%) and was controlled through the application of topical anti-glaucoma medication. Only one patient recorded $31 \mathrm{mmHg}$ once, and the pressure was controlled thereafter. One patient was hospitalized for chronic kidney disease aggravation and dropped out of the study. Other adverse events (AE) included eye discharge, subconjunctival hemorrhage, burning sensation of eye, upper respiratory infection symptom, vitreous hemorrhage, high blood pressure, gastrointestinal trouble, gastroenteritis, and tinnitus. Any progression of cataract or event of increased IOP (over $25 \mathrm{mmHg}$ ) have not affected visual acuity. (Table 3)

\section{Discussion}

This study showed that HE in DME decreased significantly during a year using DEX implant injection twice or three times over the study period. To the best of our knowledge, this is the first prospective interventional study focused on HE in DME using DEX implant for 12 months. HE area and centrally-located HE1500 area decreased continuously. Moreover, HE1500 was closely

Table 2 Univariate and multivariate analysis of factors affecting baseline HE and visual acuity

\begin{tabular}{|c|c|c|c|c|c|}
\hline \multirow{3}{*}{ Variable } & \multicolumn{2}{|c|}{${\text { Baseline } B C V A^{a}}^{a}$} & \multicolumn{3}{|c|}{ Baseline $\mathrm{HE}_{\text {area }}{ }^{\mathrm{b}}$} \\
\hline & \multirow[t]{2}{*}{ Beta } & \multirow{2}{*}{$\begin{array}{l}P \text { value } \\
\text { Univariable }\end{array}$} & \multirow[t]{2}{*}{ Beta } & \multicolumn{2}{|l|}{$P$ value } \\
\hline & & & & Univariable & Multivariable \\
\hline Male & 3.098 & 0.631 & 0.915 & 0.127 & \\
\hline Presence of SRF & -4.8 & 0.476 & 0.169 & 0.773 & \\
\hline Diffuse type ME & -6.09 & 0.319 & -0.077 & 0.708 & \\
\hline Age & -0.33 & 0.288 & 0.009 & 0.708 & \\
\hline $\mathrm{HbA1c}$ & -4.191 & $0.02^{*}$ & -0.077 & 0.746 & \\
\hline BUN & -0.755 & 0.203 & 0.007 & 0.874 & \\
\hline $\mathrm{Cr}$ & 3.856 & 0.445 & 0.284 & 0.209 & \\
\hline TG & 0.011 & 0.794 & 0.003 & 0.19 & \\
\hline Total cholesterol & 0.006 & 0.918 & 0.009 & $0.001^{*}$ & 0.165 \\
\hline $\mathrm{HDL}$ & -0.018 & 0.933 & 0 & 0.991 & \\
\hline LDL & 0.077 & 0.506 & 0.008 & $0.013^{*}$ & 0.72 \\
\hline Microaneurysm count & -0.353 & 0.116 & 0.036 & $<0.001^{*}$ & $<0.001^{*}$ \\
\hline HE area & -0.171 & 0.908 & & & \\
\hline HE1500 area & -6.839 & 0.316 & & & \\
\hline CMT & -0.025 & 0.258 & 0.002 & 0.189 & \\
\hline
\end{tabular}

BCVA best corrected visual acuity, Presence of SRF presence of macular subretinal fluid, DME diabetic macular edema, $B U N$ blood urea nitrogen, $C r$ creatinine, $T G$ triglyceride, $H D L$ high density lipoprotein, $L D L$ low density lipoprotein, $H E$ hard exudate, $H E 1500 \mathrm{HE}$ within $1500 \mu \mathrm{m}$ from the fovea, $C M T$ central macular thickness

${ }^{*} P$ value less than $0.05 ;{ }^{a}$ linear regression analysis; ${ }^{b}$ linear regression analysis after log transformation of HE area 
Table 3 Factors affecting repetitive measurements of BCVA ${ }^{a}$ for 1 year

\begin{tabular}{|c|c|c|c|}
\hline \multirow[t]{2}{*}{ Variable } & \multirow[t]{2}{*}{ Beta } & \multicolumn{2}{|l|}{$P$ value } \\
\hline & & Univariable $^{\mathbf{b}}$ & Multivariable $^{b}$ \\
\hline Male & 4.824 & 0.47 & \\
\hline Presence of SRF & -8.378 & 0.174 & \\
\hline Diffuse type DME & -2.128 & 0.729 & \\
\hline Age & -0.594 & $0.038^{*}$ & $0.011^{*}$ \\
\hline $\mathrm{HbA1c}$ & -3.842 & $0.035^{*}$ & 0.164 \\
\hline BUN & -0.583 & 0.445 & \\
\hline $\mathrm{Cr}$ & 4.354 & 0.142 & \\
\hline TG & -0.011 & 0.75 & \\
\hline Total cholesterol & 0.01 & 0.866 & \\
\hline $\mathrm{HDL}$ & 0.055 & 0.768 & \\
\hline LDL & 0.061 & 0.502 & \\
\hline Microaneurysm count & -0.382 & $0.014^{*}$ & 0.201 \\
\hline IOP increase $(>25 \mathrm{mmHg})$ & 0.212 & 0.758 & \\
\hline Cataract progression & -0.321 & 0.132 & \\
\hline HE area $^{a}$ & -0.807 & 0.383 & \\
\hline HE1500 area $^{a}$ & -4.457 & $<0.001^{*}$ & $0.004^{*}$ \\
\hline $\mathrm{CMT}^{\mathrm{a}}$ & -0.042 & $0.001^{*}$ & $<0.001^{*}$ \\
\hline
\end{tabular}

$B C V A$ best corrected visual acuity, Presence of SRF presence of macular subretinal fluid, DME diabetic macular edema, BUN blood urea nitrogen, $\mathrm{Cr}$ creatinine, $T G$ triglyceride, $H D L$ high density lipoprotein, $L D L$ low density lipoprotein, IOP intraocular pressure, $\mathrm{HE}$ hard exudate, HE1500 HE within $1500 \mu \mathrm{m}$ from the fovea, CMT central macular thickness

* $P$ value less than 0.05

${ }^{a} \mathrm{BCVA}, \mathrm{HE}$ area and HE1500 area indicates the values observed in every visit of all the study participants. Because they were clustered data of the same person, generalized estimating equation (GEE) was used as statistical analysis ${ }^{\mathrm{b}}$ The outcome variable was BCVA. The within-subject variable was the visit month. An autoregressive correlation was used as a working correlation

related to visual acuity. A distinct feature of $\mathrm{HE}$ area compared to other outcomes is its continuous regressing pattern except for the initial increase or plateau. CMT decreased after injection and reached its lowest level around 2 months after injection, at which point CMT increased until the next injection. BCVA also showed maximal improvement around 2 months after injection and then decreased. Therefore, average line plots resemble a U- or inverted U-shape for CMT and BCVA, respectively (Fig. 3). This pharmacokinetic effect of DEX implant on CMT and BCVA is consistently reported in other studies [7]. On the other hand, the current study revealed a continuous pattern of $\mathrm{HE}$ area regression. Other studies using DEX implants, which had shorter study durations or longer visit intervals than the current study, have not shown such a continuous reduction of HE $[11,12]$. HE area and HE1500 area showed increase at 1 month after first injection ( $\mathrm{p}$ value $=0.7358$ and 0.009 , respectively). This does not match to the most rapid improvement of vision and CMT at this time point. We don't know whether this is real increase of
HE because we were not able to measure the total volume through fundus photo. Abrupt resolution of macular edema and relatively lagged absorption of HE could make HE distinctively visible and this might result in increase of HE area measured using fundus photo segmentation. Relative faster resolution of fluid in central than peripheral macula might explain only significant increase of HE1500 at 1 month. This phenomenon of HE increase at early periode is also reported in monthly ranibizumab injection study [15].

The current study does not have a control group not having any intervention or undertaking different treatment. Therefore, we should be cautious when interpreting whether HE resolution is a substantial effect of DEX implant. There are several studies investigating the efficacy of intravitreal injection for the reduction of HE in DME. Secondary analysis of the BEVORDEX study investigated the efficacy of dexamethasone implant or bevacizumab on the regression of HE [11]. Both DEX implant and bevacizumab are effective at reducing $\mathrm{HE}$. However, this study suggested that DEX implant provided more rapid regression of HE from the foveal center than bevacizumab at 12 months. Another post hoc analysis was performed using the "RISE and RIDE study" [9]. This study reported that monthly ranibizumab injection resulted in significant reduction of $\mathrm{HE}$ area compared to the sham group. Total resolution of $\mathrm{HE}$ was observed in $60 \%$ of the ranibizumab group and $36 \%$ in the sham group. There are several studies which have conducted short term observation of $\mathrm{HE}$ after intravitreal injection. Monthly bevacizumab injection has been shown to not reduce $\mathrm{HE}$ area within 6 months [16]. In addition, HE count increased after three loading injections and an additional single treatment of anti-VEGF for 6 months in a separate study [17]. DEX implant and triamcinolone injection have been shown to reduce $\mathrm{HE}$, but not bevacizumab, in a 3-month observational study [12]. To summarize these studies, monthly ranizibumab was found to be superior to a "laser treatment as needed" group over a 2-year period. Monthly bevacizumab was as effective as DEX implant every 16 weeks after 2 years [11]. Specifically, DEX implant reduced HE in a short period even when the efficacy of anti-VEGF was controversial.

DEX implant is advantageous due to its injection schedule, which is relevant to economic burden and time consumption. Ranibizumab or bevacizumab were injected with fixed monthly dosing in studies suggesting efficacy on HE. However, monthly injection is not practical in ordinary clinical circumstances. Real world observational studies report 4 to 7 anti-VEGF injections per year, which is much less than monthly dosing of pivotal studies $[18,19]$. However, the average 2.7 DEX implant at 1 year observed in the present study is 
consistent with 2.4 injection per year in usual practice [20]. Thus, DEX implant for DME with HE is economically feasible and an effective method even though monthly anti-VEGF is equally effective.

The current study also showed anatomical and visual improvements. The average CMT reduction after 1 year was $89 \mu \mathrm{m}$. This reduction was less than the $187 \mu \mathrm{m}$ decrease in the BEVORDEX study which had a similar injection schedule as that of the current study; the result was rather comparable with $60 \mu \mathrm{m}$ reduction in the real world study [21, 22]. Differences in the study population can explain these discrepancies. Present study requires macular HE and is likely to include more proportion of treatment resistant patients. GEE analysis showed that HE area was related to CMT $(\beta=21.2, P=0.002)$. HE was also strongly correlated with CMT reduction in DRCR protocol I [23]. The average visual gain was 4.4 letters, and $35 \%$ of patients had a visual gain of $>10$ letters. This visual gain is comparable with the BEVORDEX study reporting 5.6 letters and 40\%, respectively [24]. Another important factor explaining current CMT and BCVA outcomes can be the elapsed time after final injection. As our last observation was performed after passing the peak efficacy of DEX implant and a third injection was performed only in 68\%, CMT and VA outcome could be underestimated at the final visit.

We could not find factors associated with changes in $\mathrm{HE}$, although there were several factors relevant to baseline HE and BCVA. Baseline HE area was related to total cholesterol and LDL concentrations. This is consistent with the results of previous reports describing that higher total cholesterol and LDL cholesterol concentrations were correlated with retinal $\operatorname{HE}[25,26]$. Baseline BCVA was correlated with lower initial HbA1c level in current study. HbA1c reflects long-term glycemic control and intensive glycemic control delays the progression of diabetic retinopathy [27]. HbA1c levels was also correlated with baseline hyperreflective foci count on OCT [28]. There is conflicting evidence of HbA1c influence on visual response to anti-VEGF treatment. HbA1c was not associated with BCVA change in 'RISE and RIDE' study, while it was associated with visual acuity improvement in 'VISTA and VIVID trials' and 'Protocol T' [29-31].

HE regressed regardless of other parameters in the current study. However, low HbA1c and low cholesterol levels showed a beneficial effect on HE reduction using the anti-VEGF treatment [17]. We may presume that the efficacy of the DEX implant on HE reduction is strong enough to overcome the influence of other factors. Or we could not find any contributing factors due to small study population.

We found that HE area was related to BCVA. In our study, baseline HE was not relevant to BCVA whether it was located centrally or not. Centrally-located HE area (HE1500) was related to BCVA in both univariable and multivariable GEE analysis, as well as CMT. HE area reduction showed weak positive correlation with BCVA improvement. The predictive value of $\mathrm{HE}$ on visual outcome is controversial. In the ETDRS study, after adjusting for other factors, foveal HE was an independent risk factor for worse VA outcome [8]. Sadda et al. showed that baseline foveal HE were associated with worse visual outcomes [15]. BEVORDEX and RISE RIDE post hoc analysis showed that HE does not affect visual outcome. DRCR.net protocol I revealed that patients having baseline HE showed better VA outcome [23]. It is not clear whether HE reduction is the reason of better visual outcome or not in protocol I. Unlike CMT which directly affected central vision, the impact of HE on vision might be limited or indirect. Older age was negatively correlated to vision in current study. Data from the RESTORE study revealed that larger treatment benefits of VA were associated with younger age,[23] suggesting that younger patients have better visual outcomes.

Corticosteroid relieves the abnormal inflammatory process and lowers VEGF level that may stabilize the barrier function of vessels [32]. Moreover, corticosteroid can stabilize the blood retinal barrier by regulating endothelial junction proteins or water channel in Muller glial cells $[33,34]$. Thus, corticosteroid might ameliorate the pathologic condition of macromolecule leakage from vessels and maintain homeostasis to help the removal of HE. Additional anti-inflammatory effects of corticosteroid might result in a faster reduction of HE compared to anti-VEGF agents.

Cataract progression and increased IOP are important $\mathrm{AE}$ of DEX implant. In the current study, we experienced $47 \%$ of patients showing cataract progression and two patients underwent cataract surgery. IOP exceeding $25 \mathrm{mmHg}$ at any time was observed in five patients (11\%) and this was relatively small portion comparing 35\% in MEAD study [7]. Except for cataract progression and increased IOP, AEs reported in this study have low clinical relevance with study drug use. In current study, cataract progression was not related to vision. It might be based upon the relative small study population or finding that cataract generally appears in the second year after intravitreal steroid therapy [35].

The present study has several limitations. First, we don't have control group to compare the effect of the study drug. Although we can infer the effect of the intervention by comparing to previous reports using other drugs, natural course data of HE in DME is limited. In addition, our study population was comprised of a relatively small number of patients, and therefore, it may not be large enough to reveal the factors influencing the HE reduction. However, the current study accomplished 
the study objective of investigating HE area change in a prospective nature.

This study clearly showed that HE in DME regressed continuously using the DEX implant except right after the first injection. We also observed visual gain and CMT reduction, which are consistent with other studies. Majority of previous studies have reported that steroid is superior in reducing HE than anti-VEGF agents within a short duration of approximately 1 year. Moreover, DEX implant schedule in the current study was an amenable treatment interval close to that in the routine clinical practice. Centrally-located HE near the fovea is related to vision. Thus, DEX implant can be considered as a proper treatment option, especially when DME is accompanied by foveal HE.

\section{Conclusions}

Dex implant was able to reduce and suppress HE of DME in generally continuous and rapid manner by injecting two or three times for a year. Dex implant improved vision and ameliorate macular edema as well. Moreover, centrally located HE around fovea was related to visual acuity. Thus, Dex implant can be a beneficial treatment option when DME accompanied by HE.

\author{
Abbreviations \\ DEX implant: Intravitreal dexamethasone implant; HE: Hard exudate; \\ DME: Diabetic macular edema; OCT: Optical coherence tomography; \\ BCVA: Best corrected visual acuity; ETDRS: Early Treatment Diabetic \\ Retinopathy Study; CMT: Central macular thickness; HE1500 area: Centrally \\ located HE area; anti-VEGF: Anti-vascular endothelial growth factor; \\ IOP: Intraocular pressure; GEE: Generalized estimating equation; SD: Standard \\ deviation; LDL: Low density lipoprotein
}

\section{Acknowledgements}

Kun Hyung Kim and Jun Hee Han provided statistical consultation to this study.

\section{Authors' contributions}

Study conceptualization: (HWK); Conduct of study (CKY, JPS, MS, SJL, JoEL, JiEL, IYC, WJJ, KYP and HYK), Data curation (CKY), Formal analysis (CKY), Writing-original draft (CKY), Writing-review \& editing (HWK, MS and JPS) All authors have read and approved the manuscript.

\section{Funding}

Allergan Korea (Seoul, Republic of Korea) provided funding and investigational drugs for this study.

The funding bodies played no role in the design of the study and collection, analysis, and interpretation of data and inwriting the manuscript.

\section{Availability of data and materials}

The data that support the findings of this study are available from the corresponding author, [H.W.K.], upon reasonable request.

\section{Ethics approval and consent to participate}

All study conduct adhered to the tenets of the Declaration of Helsinki, and the study protocol was approved by the Institutional Review Board of each site. (DAUHIRB-15-033', 129792-2015-020², 14-0224³ , 2015-02-002 ${ }^{4}, 2015-03-$ $018^{5}$, KNUH 2015-03-015 $, 91961-\mathrm{ABG}^{7}, 1503-006-040^{8}$ )

'Dong-A University Hospital, ${ }^{2}$ Inje Univertisy, Haeundae Paik hospital, ${ }^{3}$ Inje University Pusan Paik hospital, ${ }^{4}$ Gyeongsang National University Hospital,

${ }^{5}$ Yeungnam University College of Medicine, ${ }^{6}$ Kyungpook National University,
${ }^{7}$ Kosin University college of medicine, Gospel Hospital, ${ }^{8}$ College of Medicine, Pusan National University.

Written informed consent was obtained from all individual patients included in the study.

\section{CONSORT guidelines}

This study adheres to CONSORT guidelines

\section{Consent for publication}

Not applicable.

\section{Competing interests}

None of the authors has any financial/conflicting interests to disclose.

\section{Author details}

${ }^{1}$ Department of Ophthalmology, Seoul National University Hospital, Seoul, Korea. ${ }^{2}$ Department of Ophthalmology, Yeungnam University College of Medicine, Daegu, Korea. ${ }^{3}$ Department of Ophthalmology, School of Medicine, Kyungpook National University, Daegu, Korea. ${ }^{4}$ Department of Ophthalmology, Kosin University college of medicine, Gospel Hospital, Busan, Korea. ${ }^{5} \mathrm{~J}$ Eye Center, Busan, Korea. ${ }^{6}$ College of Medicine, Pusan National University, Yangsan, Korea. ${ }^{7}$ Institute of Health Sciences, Gyeongsang National University Hospital, Gyeongsang National University, Jinju, Korea. ${ }^{8}$ Dong-A University Hospital, Busan, Korea. ${ }^{9}$ Inje Univertisy, Haeundae Paik hospital, 875, Haeun-daero, Haeundae-gu, 48108 Busan, Korea. ${ }^{10}$ Inje University Pusan Paik hospital, 875, Haeun-daero, Haeundae-gu, 48108 Busan, Korea.

Received: 19 August 2020 Accepted: 21 December 2020 Published online: 15 January 2021

\section{References}

1. Yau JWYY, Rogers SL, Kawasaki R, Lamoureux EL, Kowalski JW, Bek T, et al. Global prevalence and major risk factors of diabetic retinopathy. Diabetes Care. 2012;35:556-64. doi:https://doi.org/10.2337/dc11-1909.

2. Klein R, Klein BE, Moss SE, Cruickshanks KJ. The Wisconsin Epidemiologic Study of Diabetic Retinopathy. XV. The long-term incidence of macular edema. Ophthalmology. 1995;102:7-16. http://www.ncbi.nlm.nih.gov/ pubmed/7831044. Accessed 13 Nov 2018.

3. Aiello LM, Ferris FL. Photocoagulation for Diabetic Macular Edema. Arch Ophthalmol. 1987;105:1163. doi:https://doi.org/10.1001/archopht.1987. 01060090021004.

4. Heier JS, Korobelnik J-F, Brown DM, Schmidt-Erfurth U, Do DV, Midena E, et al. Intravitreal Aflibercept for Diabetic Macular Edema: 148-Week Results from the VISTA and VIVID Studies. Ophthalmology. 2016;123:2376-85. doi: https://doi.org/10.1016/j.ophtha.2016.07.032

5. Elman MJ, Aiello LP, Beck RW, Bressler NM, Bressler SB, Edwards AR, et al. Randomized Trial Evaluating Ranibizumab Plus Prompt or Deferred Laser or Triamcinolone Plus Prompt Laser for Diabetic Macular Edema. Ophthalmology. 2010;117:1064-77.e35. doi:https://doi.org/10.1016/j.ophtha. 2010.02.031.

6. Brown DM, Nguyen QD, Marcus DM, Boyer DS, Patel S, Feiner L, et al. Longterm outcomes of ranibizumab therapy for diabetic macular edema: The 36month results from two phase III trials: RISE and RIDE. Ophthalmology. 2013; 120:2013-22. doi:https://doi.org/10.1016/j.ophtha.2013.02.034.

7. Boyer DS, Yoon YH, Belfort R, Bandello F, Maturi RK, Augustin AJ, et al. Three-year, randomized, sham-controlled trial of dexamethasone intravitreal implant in patients with diabetic macular edema. Ophthalmology. 2014;121: 1904-14. doi:https://doi.org/10.1016/j.ophtha.2014.04.024.

8. Chew EY, Klein ML, Ferris FL, Remaley NA, Murphy RP, Chantry K, et al. Association of elevated serum lipid levels with retinal hard exudate in diabetic retinopathy. Early Treatment Diabetic Retinopathy Study (ETDRS) Report 22. Arch Ophthalmol (Chicago, III 1960). 1996;114:1079-84. http:// www.ncbi.nlm.nih.gov/pubmed/8790092. Accessed 22 Jun 2018.

9. Domalpally A, Ip MS, Ehrlich JS. Effects of intravitreal ranibizumab on retinal hard exudate in diabetic macular edema: findings from the RIDE and RISE phase III clinical trials. Ophthalmology. 2015;122:779-86. doi:https://doi.org/ 10.1016/j.ophtha.2014.10.028.

10. Otani T, Kishi S. Tomographic findings of foveal hard exudates in diabetic macular edema. Am J Ophthalmol. 2001;131:50-4. http://www.ncbi.nlm.nih. gov/pubmed/11162979. Accessed 14 Nov 2018. 
11. Mehta H, Fraser-Bell S, Yeung A, Campain A, Lim LL, Quin GJ, et al. Efficacy of dexamethasone versus bevacizumab on regression of hard exudates in diabetic maculopathy: Data from the BEVORDEX randomised clinical trial. $\mathrm{Br}$ J Ophthalmol. 2016;100:1000-4. doi:https://doi.org/10.1136/bjophthalmol2015-307797.

12. Shin YU, Hong EH, Lim HW, Kang MH, Seong M, Cho H. Quantitative evaluation of hard exudates in diabetic macular edema after short-term intravitreal triamcinolone, dexamethasone implant or bevacizumab injections. BMC Ophthalmol. 2017;17:1-9.

13. Kim BY, Smith SD, Kaiser PK. Optical Coherence Tomographic Patterns of Diabetic Macular Edema. Am J Ophthalmol. 2006;142:405-12.e1. doi:https:// doi.org/10.1016/j.ajo.2006.04.023.

14. Cole ED, Onal S, Belfort R, Kniggendorf VF, Regatieri CV, Duker JS, et al. Comparison of Optical Coherence Tomography Angiography and Fluorescein Angiography for the Identification of Retinal Vascular Changes in Eyes With Diabetic Macular Edema. Ophthalmic Surgery, Lasers Imaging Retin. 2016;47:1013-9.

15. Srinivas S, Verma A, Nittala MG, Alagorie AR, Nassisi M, Gasperini J, et al. Effect of Intravitreal Ranibizumab on Intraretinal Hard Exudates in Eyes with Diabetic Macular Edema. Am J Ophthalmol. 2020;211:183-90. doi:https://doi. org/10.1016/j.ajo.2019.11.014

16. Jeon S, Lee WK. Effect of intravitreal bevacizumab on diabetic macular edema with hard exudates. Clin Ophthalmol. 2014;8:1479-86.

17. Pemp B, Deák G, Prager S, Mitsch C, Lammer J, Schmidinger G, et al. Distribution of intraretinal exudates in diabetic macular edema during antivascular endothelial growth factor therapy observed by spectral domain optical coherence tomography and fundus photography. Retina. 2014;34: 2407-15. doi:https://doi.org/10.1097/IAE.0000000000000250.

18. Patrao NV, Antao S, Egan C, Omar A, Hamilton R, Hykin PG, et al. Real-World Outcomes of Ranibizumab Treatment for Diabetic Macular Edema in a United Kingdom National Health Service Setting. Am J Ophthalmol. 2016; 172:51-7. doi:https://doi.org/10.1016/j.ajo.2016.09.002.

19. Dugel PU, Layton A, Varma RB. Diabetic Macular Edema Diagnosis and Treatment in the Real World: An Analysis of Medicare Claims Data (2008 to 2010). Ophthalmic Surg Lasers Imaging Retina. 2016;47:258-67. https://doi. org/10.3928/23258160-20160229-09.

20. Querques G, Darvizeh F, Querques L, Capuano V, Bandello F, Souied EH. Assessment of the Real-Life Usage of Intravitreal Dexamethasone Implant in the Treatment of Chronic Diabetic Macular Edema in France. J Ocul Pharmacol Ther. 2016;32:383-9. doi:https://doi.org/10.1089/jop.2016.0010.

21. Singer MA, Dugel PU, Fine HF, Capone A, Maltman J. Real-World Assessment of Dexamethasone Intravitreal Implant in DME: Findings of the Prospective, Multicenter REINFORCE Study. Ophthalmic Surgery. Lasers Imaging Retin. 2018;49:425-35. doi:https://doi.org/10.3928/2325816020180601-07.

22. Fraser-Bell S, Lim LL, Campain A, Mehta H, Aroney C, Bryant J, et al. Bevacizumab or Dexamethasone Implants for DME: 2-year Results (The BEVORDEX Study). Ophthalmology. 2016;123:1399-401. doi:https://doi.org/ 10.1016/j.ophtha.2015.12.012.

23. Bressler SB, Qin H, Beck RW, Chalam KV, Kim JE, Melia M, et al. Factors associated with changes in visual acuity and central subfield thickness at 1 year after treatment for diabetic macular edema with ranibizumab. Arch Ophthalmol. 2012;130:1153-61. doi:https://doi.org/10.1001/archophthalmol. 2012.1107.

24. Gillies MC, Lim LL, Campain A, Quin GJ, Salem W, Li J, et al. A randomized clinical trial of intravitreal bevacizumab versus intravitreal dexamethasone for diabetic macular edema: The BEVORDEX study. Ophthalmology. 2014; 121:2473-81.

25. Davoudi S, Papavasileiou E, Roohipoor R, Cho H, Kudrimoti S, Hancock H, et al. Optical coherence tomography characteristics of macular edema and hard exudates and their association with lipid serum levels in type 2 diabetes. Retina. 2016;36:1622-9.

26. Sasaki M, Kawasaki R, Noonan JE, Wong TY, Lamoureux E, Wang JJ. Quantitative measurement of hard exudates in patients with diabetes and their associations with serum lipid levels. Investig Ophthalmol Vis Sci. 2013; 54:5544-9.

27. Diabetes Control and Complications Trial Research Group. Nathan DM, Genuth S, Lachin J, Cleary P, Crofford O, et al. The effect of intensive treatment of diabetes on the development and progression of long-term complications in insulin-dependent diabetes mellitus. N Engl J Med. 1993; 329:977-86. doi:https://doi.org/10.1056/NEJM199309303291401.
28. Framme C, Schweizer $P$, Imesch M, Wolf S, Wolf-Schnurrbusch U. Behavior of SD-OCT-detected hyperreflective foci in the retina of anti-VEGF-treated patients with diabetic macular edema. Invest Ophthalmol Vis Sci. 2012;53: 5814-8. doi:https://doi.org/10.1167/iovs.12-9950.

29. Singh RP, Habbu K, Ehlers JP, Lansang MC, Hill L, Stoilov I. The Impact of Systemic Factors on Clinical Response to Ranibizumab for Diabetic Macular Edema. Ophthalmology. 2016;123:1581-7. doi:https://doi.org/10.1016/j. ophtha.2016.03.038.

30. Bressler SB, Odia I, Maguire MG, Dhoot DS, Glassman AR, Jampol LM, et al. Factors Associated with Visual Acuity and Central Subfield Thickness Changes When Treating Diabetic Macular Edema with Anti-Vascular Endothelial Growth Factor Therapy: An Exploratory Analysis of the Protocol T Randomized Clinical Trial. JAMA Ophthalmol. 2019;137:382-9. doi:https:// doi.org/10.1001/jamaophthalmol.2018.6786.

31. Singh RP, Wykoff CC, Brown DM, Larsen M, Terasaki H, Silva FQ, et al. Outcomes of Diabetic Macular Edema Patients by Baseline Hemoglobin A1c: Analyses from VISTA and VIVID. Ophthalmol Retin. 2017;1:382-8. doi: https://doi.org/10.1016/j.oret.2017.02.003.

32. Sohn HJ, Han DH, Kim IT, Oh IK, Kim KH, Lee DY, et al. Changes in aqueous concentrations of various cytokines after intravitreal triamcinolone versus bevacizumab for diabetic macular edema. Am J Ophthalmol. 2011;152:686-94.

33. Zhao $M$, Bousquet $E$, Valamanesh $F$, Farman $N$, Jeanny J-C, Jaisser $F$, et al. Differential regulations of AQP4 and Kir4.1 by triamcinolone acetonide and dexamethasone in the healthy and inflamed retina. Invest Ophthalmol Vis Sci. 2011;52:6340-7. doi:https://doi.org/10.1167/iovs.11-7675.

34. Keil JM, Liu X, Antonetti DA. Glucocorticoid induction of occludin expression and endothelial barrier requires transcription factor p54 NONO. Invest Ophthalmol Vis Sci. 2013;54:4007-15. doi:https://doi.org/10.1167/iovs. 13-11980.

35. Gillies MC, Sutter FKP, Simpson JM, Larsson J, Ali H, Zhu M. Intravitreal Triamcinolone for Refractory Diabetic Macular Edema. Two-Year Results of a Double-Masked, Placebo-Controlled, Randomized Clinical Trial. Ophthalmology. 2006;113:1533-8. doi:https://doi.org/10.1016/j.ophtha.2006. 02.065

\section{Publisher's Note}

Springer Nature remains neutral with regard to jurisdictional claims in published maps and institutional affiliations.
Ready to submit your research? Choose BMC and benefit from:
- fast, convenient online submission
- thorough peer review by experienced researchers in your field
- rapid publication on acceptance
- support for research data, including large and complex data types
- gold Open Access which fosters wider collaboration and increased citations
- maximum visibility for your research: over $100 \mathrm{M}$ website views per year
At $\mathrm{BMC}$, research is always in progress. 